\title{
2D-Omnidirectional Hard-X-Ray Scattering Sensitivity in a Single Shot
}

\author{
Matias Kagias, Zhentian Wang, Pablo Villanueva-Perez, Konstantins Jefimovs, and Marco Stampanoni* \\ Swiss Light Source, Paul Scherrer Institute, 5232 Villigen, Switzerland \\ Institute for Biomedical Engineering, University and ETH Zurich, 8092 Zurich, Switzerland
}

(Received 17 December 2015; published 3 March 2016)

\begin{abstract}
$\mathrm{X}$-ray scattering imaging can provide complementary information to conventional absorption based radiographic imaging about the unresolved microstructures of a sample. The scattering signal can be accessed with various methods based on coherent illumination, which span from self-imaging to speckle scanning. The directional sensitivity of the existing real space imaging methods is limited to a few directions on the imaging plane and requires scanning of the optical components, or the rotation of either the sample or the imaging setup, in order to cover the full range of possible scattering directions. In this Letter the authors propose a new method that allows the simultaneous acquisition of scattering images in all possible directions in a single shot. This is achieved by a specialized phase grating and a detector with sufficient spatial resolution to record the generated interference fringe. The structural length scale sensitivity of the system can be tuned by varying its geometry for a fixed grating design. Taking into account ongoing developments in the field of compact x-ray sources that allow high brightness and sufficient spatial coherence, the applicability of omnidirectional scattering imaging in industrial and medical settings is boosted significantly.
\end{abstract}

DOI: 10.1103/PhysRevLett.116.093902

X-ray grating interferometry (GI) can provide simultaneously three complementary contrasts: absorption, differential phase, and small-angle scattering [1-3] also known as dark-field. The phase signal is highly sensitive to the electron density variations in the sample and can reveal differences between materials with similar absorption properties. The dark-field signal is able to access unresolved structural variations of the sample in the (sub)micrometer scale, which is beyond the resolution capability of the imaging modality. It has been demonstrated that both differential phase and darkfield signals can provide valuable information additional to the conventional absorption contrast in medical imaging [4-6], material science, and nondestructive testing [7]. Especially, the dark-field signal has drawn great attention due to its success in providing quantitative or inaccessible structural information in radiographic applications [8-12].

In general, the dark-field signal exhibits highly directional behavior if the underlying microstructure of the sample is anisotropic. However, up to now GI has mainly been composed of linear gratings and the dark-field sensitivity is therefore only perpendicular to the grating lines. To obtain multiple-direction dark-field sensitivity, either the sample or the interferometer needs to be rotated [13-14], which is a time-consuming procedure. The usage of 2D gratings can mitigate the issue but can only provide dark-field sensitivity in up to four directions. Moreover, the noise performance is not the same in all directions due to the different modulation orders of the phase stepping curves [15]. An alternative speckle scanning technique [16-17] is proposed to sense the dark-field signal by scanning a membrane in the direction of interest; however, only two directions can be obtained. To cope with samples containing structures with an unknown orientation, it would be favorable to achieve an omnidirectional dark-field sensitivity with a straightforward imaging setup, without requiring motion of the sample or the optical elements. This would ensure that all microstructures, highly oriented or not, can be detected. In this work, we propose a single-shot imaging method with 2D-ominidirectional dark-field sensitivity using a grating design consisting of a $2 \mathrm{D}$ repetition of a small pitch circular grating. To achieve single-shot imaging, the self-image of the grating is recorded with sufficient spatial resolution. Local fringe analysis is then performed to obtain the dark-field signal of the sample in all directions of the imaging plane. In addition, the differential phase contrast (DPC) in the vertical and horizontal directions can also be retrieved.

The proposed grating can be understood as a $2 \mathrm{D}$ periodic repetition of a unit cell; a schematic representation is given in Fig. 1(a). In order to obtain equal sensitivity in all directions of the imaging plane a periodic shape with a circular symmetry is necessary. The only geometrical shape conforming to this requirement is a circular grating. The design is characterized by two periodicities: a global one $P$, which is the repetition rate of a unit cell, and a local one $g_{1}$, which is the period within the unit cell. Optimum coverage of the full field of view (FOV) can be achieved when the aforementioned periodicities follow the relation $P=N g_{1}$, $N \in \mathbb{N}$. The main assumptions for extracting meaningful information from such an imaging setup are that the sample properties, i.e., the absorption, phase, and scattering distributions, are single valued within one repetition of the 


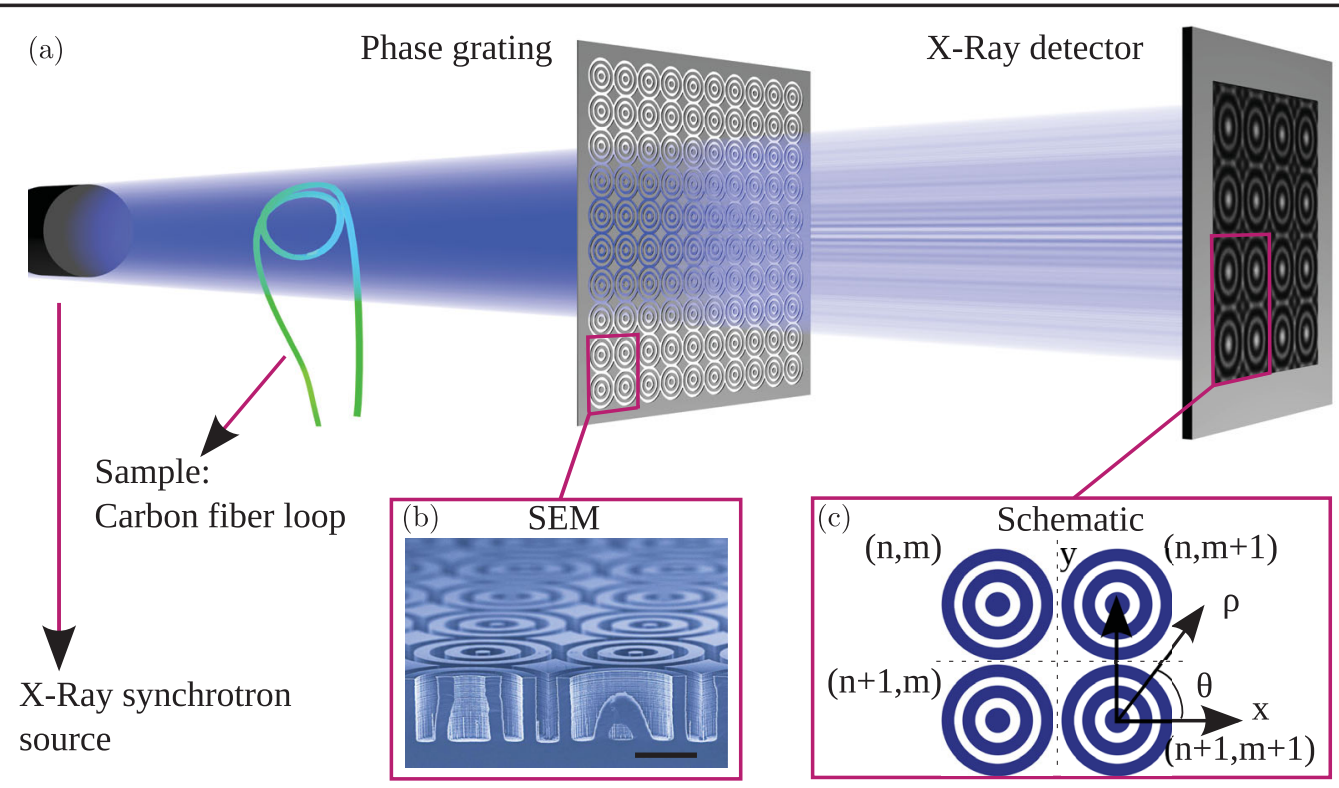

FIG. 1. Schematic of experimental setup. From left to right: the source of coherent x-rays, the carbon fiber loop used as the sample, the phase grating accompanied by the SEM image (b) of the fabricated grating (scale bar: $10 \mu \mathrm{m}$ ), and the x-ray detector with the projected experimental interference fringe at $17.14 \mathrm{~cm}$ with an effective pixel size of $0.65 \mu \mathrm{m}$. A schematic (c) of the recorded fringe is also presented to explain the local coordinates regarding the final images.

unit cell. The unit cell periodicity $P$ will define the pixel size of the final image with which the directional dark-field DPC in the $x$ and $y$ directions and the absorption signals will be reconstructed. Hence, $P$ has to be chosen appropriately according to the desired spatial resolution and sample properties. The period of the circular grating $g_{1}$ will define the dark-field sensitivity but should be large enough in order to be recorded directly by the detector in use. The detector is placed at the Talbot distance [1], which for a parallel beam geometry and a $\pi / 2$ shift grating is given by

$$
D_{m}=m \frac{g_{1}^{2}}{2 \lambda},
$$

where $m=1,2,3, \ldots, g_{1}$ is the period of the circular unit cell and $\lambda$ is the $\mathrm{x}$-ray wavelength. The generated image at this distance will not be a perfect self-image due to the finite number of periods contained in one unit cell. This will result in border effects between consecutive unit cells. This effect is mostly noticeable in the diagonal direction. However, due to the finite coherence length of the incoming beam these effects are mitigated and a reasonable approximation of the self-image can be observed as seen in Fig. 1(a). In conventional grating interferometry the dark-field signal is extracted from the observed visibility reduction of the generated interference fringe due to the sample. A broadening of the angular distribution of the incoming beam will be introduced due to small angle scattering. This broadening will smear out the interference fringe and cause visibility reduction. In the case of a circular grating and constant sample properties within the area of the grating, the detection of the visibility reduction along different radii is equivalent to the retrieval of directional dark-field images of the sample under all possible angles defined on the imaging plane.
In general the sample under examination can be described by the spatial variations of the refractive index; the fine spatial variations cause small angle scattering. On the other hand the resolvable variations cause refraction of the incoming beam, which will introduce a shift of the location of the interference fringe [18]. For the proposed grating design this means a shift on the imaging plane of the local circular gratings. By measuring this shift DPC images in the $x$ and $y$ direction can be extracted.

A standard experiment requires the acquisition of two images: a flat image with only a grating and a sample image with both a grating and sample in the beam. It is assumed that the spatial resolution capability of the detector is sufficient to record the generated interference fringe. Once the flat image has been recorded a unit cell finding algorithm is used to detect each unit cell since they are analyzed individually. Because of the circular nature of the unit cells they exhibit a clear maximum of intensity at the center point [19]; this maximum is used as a detection criterion of the unit cells. Once the centers have been detected, the square area around each center corresponding to the projected size of the grating unit cell is cropped. The recorded flat self-image of the unit cell $(n, m)$ can be approximated with the following equation:

$$
I_{f}^{(n, m)}(x, y) \approx A_{f}^{(n, m)}+B_{f}^{(n, m)}(\theta) \cos \left(2 \pi \frac{\sqrt{x^{2}+y^{2}}}{g_{1}}\right),
$$

where $x, y$ are the local Cartesian coordinates at each unit cell $(n, m)$ with the origin being defined as the center of the unit cell, $\theta$ is the angle $\arctan (y / x), A_{f}(n, m)$ denotes the average intensity in the defined area, and $B_{f}(n, m, \theta)$ is the 
angular dependent scattering coefficient. The annotations concerning the local coordinate systems of each unit cell are summarized in Fig. 1(c). When the sample is introduced in the beam the fringe at each unit cell will be

$$
\begin{aligned}
& I_{s}^{(n, m)}(x, y) \\
& \quad \approx A_{s}^{(n, m)}+B_{s}^{(n, m)}\left(\theta^{\prime}\right) \cos \left(2 \pi \frac{\sqrt{\left(x-x_{0}\right)^{2}+\left(y-y_{0}\right)^{2}}}{g_{1}}\right),
\end{aligned}
$$

where

$$
\theta^{\prime}=\arctan \left(\frac{y-y_{0}}{x-x_{0}}\right)
$$

and $\left(x_{0}, y_{0}\right)$ corresponds to the shift of each unit cell due to the refraction of the sample. The transmission contrast can be obtained in a straightforward manner by calculating the ratio of the average sample and flat measurements at each unit cell

$$
T(n, m)=\frac{\sum_{x} \sum_{y} I_{s}^{(n, m)}(x, y)}{\sum_{x} \sum_{y} I_{f}^{(n, m)}(x, y)}=\frac{A_{s}^{(n, m)}}{A_{f}^{(n, m)}} .
$$

Before calculating the dark-field signal both the flat and sample measurements are transformed from Cartesian to polar coordinates. The dark-field signal at angle $\phi$ is calculated by using Fourier analysis in a similar manner to standard grating interferometry. Specifically, the discrete Fourier transform $R_{k}$ of the line passing through the center of the unit cell at each angle $\phi$ is calculated; the dark-field signal at that angle is then given from the ratio of the $N$ th harmonic. In more detail each directional image is obtained from the following equation:

$$
C(n, m, \phi)=\frac{R_{N}^{s} R_{0}^{f}}{R_{N}^{f} R_{0}^{s}}=\frac{B_{s}^{(n, m)}\left(\theta^{\prime}=\phi\right) A_{f}^{(n, m)}}{B_{f}^{(n, m)}(\theta=\phi) A_{s}^{(n, m)}} .
$$

The angular resolution of the dark-field images is mainly dictated by the number of pixels contained in one unit cell and the spatial resolution of the detector. Another factor that comes into play is the interpolation method used for converting the Cartesian sampled fringe into discrete polar coordinates. In the worst case of a nearest neighbor interpolation the number of scattering images one can obtain is $M \pi / 2$, where $M$ is the number of sampling points along the $x$ and $y$ directions. Therefore, the supremum of the angular resolution is $2 / M \mathrm{rad}$. The phase shift introduced by the refraction caused by the sample can be extracted from the displacement $\left(x_{0}, y_{0}\right)$. The analytical signal along $x$ or $y$ is calculated for both the flat and sample measurements by means of the Hilbert transform. The analytical signals can be used to extract the local phase difference of the fringes as described in Ref. [20]. Theoretically, this local phase difference, when expressed in polar coordinates, is given by

$$
\Phi\left(\rho, \theta, \theta_{0}, \rho_{0}\right)=\frac{2 \pi}{g_{1}}\left[\sqrt{\rho^{2}+\rho_{0}^{2}-2 \rho \rho_{0} \cos \left(\theta-\theta_{0}\right)}-\rho\right]
$$

The detailed calculation of this local phase shift is given in the Supplemental Material [21]. Least mean square fitting can be utilized to estimate the shift $\left(\rho_{0}, \theta_{0}\right)$ and in turn $\left(x_{0}, y_{0}\right)$. The phase shift can also be extracted by other methods based on the cross correlation of the two fringes like spatial mapping [22-23].

The validity of the proposed technique was demonstrated with experimental measurements performed at the TOMCAT beam line [24], Swiss Light Source, Switzerland. The experimental setup is summarized in Fig. 1(a). A phase shifting grating with a radial period of $5 \mu \mathrm{m}$ and unit cell period of $25 \mu \mathrm{m}$ was fabricated in house by $e$-beam lithography and deep reactive ion etching of $\mathrm{Si}$. The grating was etched to a depth of $10.92 \mu \mathrm{m}$, which at $17 \mathrm{keV}$ illumination produces a phase shift of $\pi / 2$. A scanning electron microscopy (SEM) image of the fabricated grating can be seen in Fig. 1(b). The grating was placed $25 \mathrm{~m}$ from the source; the distance between the grating and the detector was set to be equal to the first fractional Talbot distance for the given energy and grating pitch, which was equal to $17.14 \mathrm{~cm}$. The sample was always placed prior to the phase grating and as close as possible to it. The photon energy was selected by a Si [111] monochromator. The $\mathrm{x}$ rays were converted to visible light with a $20 \mu \mathrm{m}$ thick LuAG:Ce scintillator (lutetium aluminum garnet activated by cerium) and then recorded by a pco.edge $4.2 \mathrm{CCD}$ camera with a tenfold magnification. The tenfold magnification resulted in an effective pixel size of $0.65 \mu \mathrm{m}$, which allowed the successful recording of the interference fringe. Part of the experimental fringe on the detector can be seen in Fig. 1(a). However, at this magnification the FOV is limited to $1.2 \mathrm{~mm}$; therefore, multiple scans were performed in order to image samples of larger dimensions, and the final images were composed by stitching together the individual images from each scan. For the proof of principle a loop of carbon fibers was selected as a validation sample. The dark-field signal from the fibers depends on their orientation; therefore, the loop will produce scattering covering all the angular directions on the imaging plane. The exposure time for each full FOV acquisition was $10 \mathrm{~s}$; flat field measurements were performed in between each scan in order to minimize background variations due to potential instabilities of the beam and optical components. The total acquisition time was mainly dictated by the slow motion of the linear stage that moves the sample in and out from the beam to acquire the flat field measurements and the low flux of the Si [111] monochromator.

A total number of 25 directional dark-field images covering the range $[0, \pi]$ were calculated. The conversion from Cartersian to polar coordinates was done by bicubic interpolation. The individual scattering images are shown in a video in the Supplemental Material [21]. To avoid displaying all the individual scattering images, an image representing the most prominent scattering direction is composed as proposed 


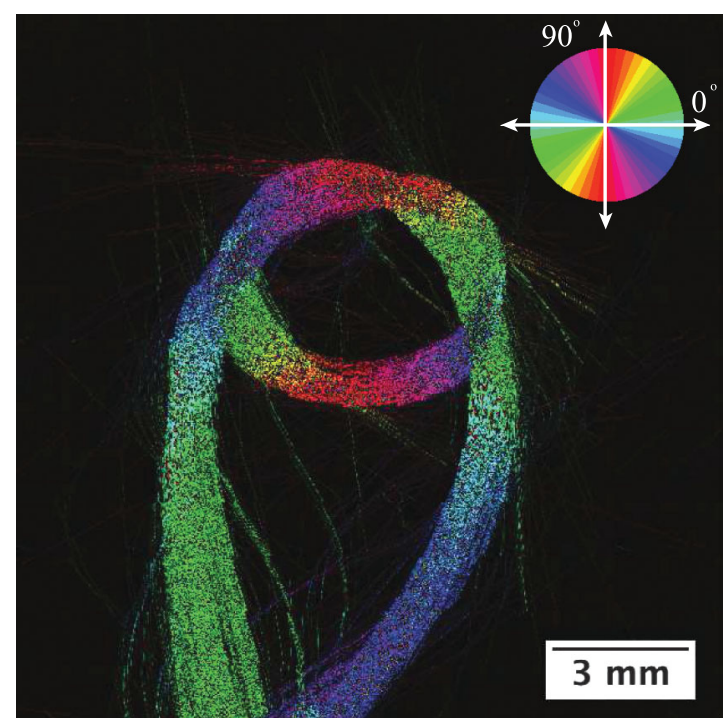

FIG. 2. Multidirectional scattering image of a carbon fiber loop; the orientation of the fibers is modulated with the color encoding the strongest scattering direction at each pixel.

in previous publications [25]. At each pixel the angular scattering distribution can be fitted by a cosine as described in Ref. [25]. The validity of this model is limited to scattering systems exhibiting a single preferred scattering direction. The average value describes the mean scattering strength of the corresponding location of the sample; the amplitude is characteristic of the directionality of the scattering and the phase of the fitted cosine defines the strongest scattering angle. The directional scattering information, including the scattering strength and the angle, is encoded into a single image using the hue saturation value (HSV) color space. More specifically, the hue corresponds to the scattering angle; the saturation is set to 1 and the value is defined as the normalized amplitude of the scattering directionality in order to fill the span $[0,1]$, meaning that dark areas in the image correspond to areas with no directional scattering. In Fig. 2 the directional

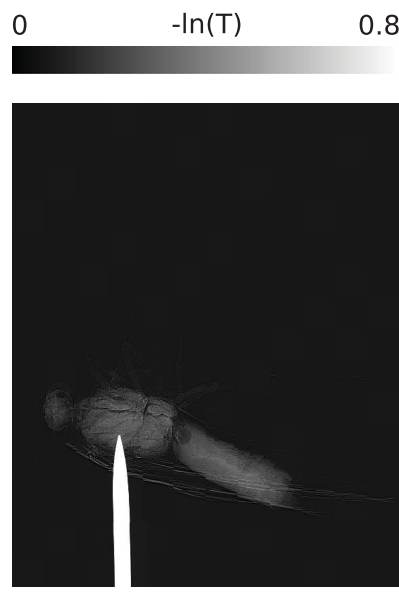

(a)

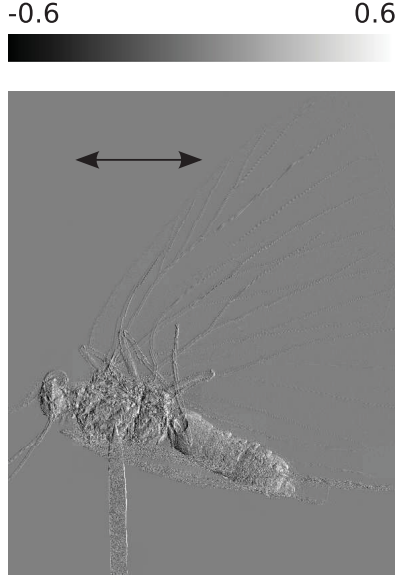

(b) scattering image of a carbon fiber loop is shown. The color of the loop changes according to the direction caused by the orientation of the fibers. The absorption and DPC images are included in the Supplemental Material [21]. A second, biological sample was also scanned with the same imaging parameters as the carbon fiber loop. The selected sample was a butterfly fixed on the tip of a steel needle. The corresponding images are presented in Figs. 3 with Fig. 3(a) being the transmission image, Fig. 3(b) the DPC in the $x$ direction, Fig. 3(c) the DPC in the $y$ direction, and Fig. 3(d) the directional scattering image. The butterfly exhibits a number of interesting features. The body of the butterfly has strong scattering characteristics but not a strong directionality; the wings on the other hand exhibit highly directional scattering of various intensities. These strongly scattering structures of the wings are mainly the veins, while weaker scattering can be observed from the scales of the wings; the signal strength was limited due to the low thickness of the wing. The second wing of the butterfly was roughly aligned with the beam; this resulted in a higher projected thickness and in turn to a high scattering signal. Other anatomical features that are clearly visible in the directional scattering image are the antennas and legs of the butterfly. The size range of the structures that the current system is sensitive to can be related to the so called autocorrelation length $[18,26]$, which is given by $d=\lambda z / g_{1}$, where $\lambda$ is the $\mathrm{x}$-ray wavelength and $z$ is the distance between the sample and the detector. For the given configuration the autocorrelation length is $2.5 \mu \mathrm{m}$. However, by tuning the imaging distance different length scales can be sensed.

The main principle of detecting the visibility reduction in all directions is based on the capability of recording the generated interference fringe directly. This allows a singleshot acquisition scheme and simultaneously removes the need for absorption gratings that with the current technologies exhibit major fabrication challenges [27-28]. At the moment appropriate optics are used in order to achieve the necessary resolution to resolve the fringe; however, recent

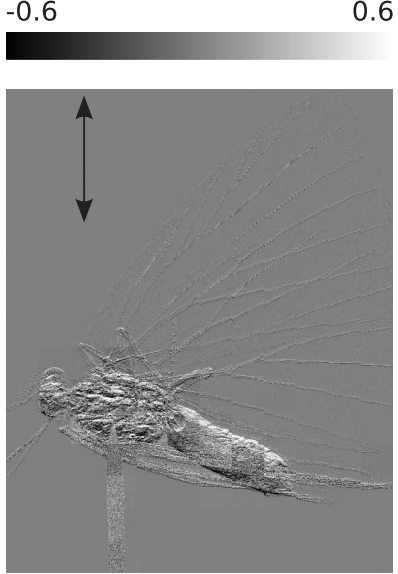

(c)

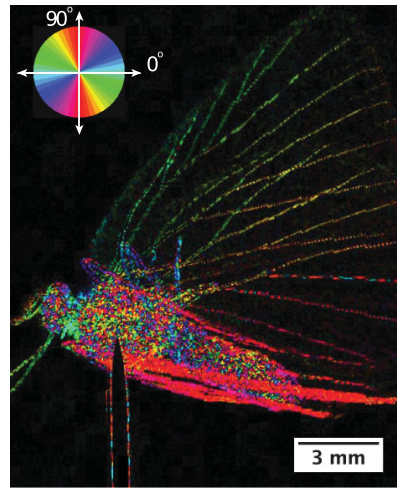

(d)

FIG. 3. (a) Absorption, (b) differential phase along $x$, (c) differential phase along $y$, and (d) directional scattering image of a butterfly fixed on a steel needle. The extracted scattering orientations are related to structures in the size range of the autocorrelation length of the system, which in our case is $2.5 \mu \mathrm{m}$. 
developments in detector research have made possible resolution enhancement beyond the pixel size of charge integrating hybrid detectors with single photon sensitivity [29]. Combining such detectors with omnidirectional sensitive interferometry might result in a highly promising approach to low-dose, high-sensitive imaging.

To summarize, the authors have presented an interferometer functioning in a single-shot mode with the following characteristics: omnidirectional dark-field sensitivity covering all possible directions on the imaging plane, differential phase contrast images in both the $x$ and $y$ directions, and absorption contrast. This is achieved with a dedicated grating design composed of local circular gratings that are repeated in a mosaic manner. No rotation or scanning of the sample or the gratings is necessary during acquisition compared to existing methods. The angular sensitivity is dictated by the repetition rate of the unit cell and the spatial resolution of the detector in use. The feasibility of the method was demonstrated by imaging a sample with a predictable behavior in a beam line setting. A biological sample was also examined in order to demonstrate the performance of the method on realistic structures. The proposed method grossly simplifies the access to multidirectional dark-field information; this is accomplished by transforming what used to be a complicated experimental setup into a single-shot-single-grating experiment. This simplification in combination with highly bright and coherent compact $\mathrm{x}$-ray sources can make GI with omnidirectional scattering sensitivity applicable in medical and industrial settings.

Part of this work has been supported by ERC Grant No. ERC-2012-StG 310005-PhaseX.

*Corresponding author. marco.stampanoni@psi.ch

[1] T. Weitkamp, A. Diaz, C. David, F. Pfeiffer, M. Stampanoni, P. Cloetens, and E. Ziegler, Opt. Express 13, 6296 (2005).

[2] A. Momose, S. Kawamoto, I. Koyama, Y. Hamaishi, K. Takai, and Y. Suzuki, Jpn. J. Appl. Phys. 42, L866 (2003).

[3] C. David, B. Nöhammer, H. H. Solak, and E. Ziegler, Appl. Phys. Lett. 81, 3287 (2002).

[4] M. Stampanoni, Z. Wang, T. Thüring, C. David, E. Roessl, M. Trippel, R. A. Kubic-Huch, G. Singer, M. K. Kohl, and N. Hauser, Investigative Radiology 46, 801 (2011).

[5] D. Stutman, T. J. Beck, J. A. Carrino, and C. O. Bingham, Phys. Med. Biol. 56, 5697 (2011).

[6] T. Thüring, R. Guggenberger, H. Alkadhi, J. Hodler, M. Vich, Z. Wang, C. David, and M. Stampanoni, Skeletal Radiol. 42, 827 (2013).

[7] V. Revol, I. Jerjen, C. Kottler, P. Schütz, R. Kaufmann, T. Lüthi, U. Sennhauser, U. Straumann, and C. Urban, J. Appl. Phys. 110, 044912 (2011).

[8] Z. Wang and M. Stampanoni, Phys. Med. Biol. 60, 4123 (2015)
[9] Z. Wang, N. Hauser, G. Singer, M. Trippel, R. A. Kubik-Huch, C. W. Schneider, and M. Stampanoni, Nat. Commun. 5, 3797 (2013).

[10] M. Bech, A. Tapfer, A. Velroyen, A. Yaroshenko, B. Pauwels, J. Hostens, P. Bruyndonckx, A. Sasov, and F. Pfeiffer, Sci. Rep. 3, 3209 (2013).

[11] S. Schleede, F. G. Meinel, M. Bech, J. Herzen, K. Achterhold, G. Potdevin, A. Malecki, S. Adam-Neumair, S. F. Thieme, F. Bamberg, K. Nikolaou, A. Bohla, A. Ö. Yildirim, R. Loewen, M. Gifford, R. Ruth, O. Eickelberg, M. Reiser, and F. Pfeiffer, Proc. Natl. Acad. Sci. U.S.A. 109, 17880 (2012).

[12] K. Hellbach, A. Yaroshenko, F. G. Meinel, A. Ö. Yildirim, T. M. Conlon, M. Bech, M. Mueller, A. Velroyen, M. Notohamiprodjo, F. Bamberg, S. Auweter, M. Reiser, O. Eickelberg, and F. Pfeiffer, Investigative Radiology 50, 430 (2015).

[13] T. H. Jensen, M. Bech, I. Zanette, T. Weitkamp, C. David, H. Deyhle, S. Rutishauser, E. Reznikova, J. Mohr, R. Feidenhans'l, and F. Pfeiffer, Phys. Rev. B 82, 214103 (2010).

[14] J. Vogel, F. Schaff, A. Fehringer, C. Jud, M. Wieczorek, F. Pfeiffer, and T. Lasser, Opt. Express 23, 15134 (2015).

[15] I. Zanette, T. Weitkamp, T. Donath, S. Rutishauser, and C. David, Phys. Rev. Lett. 105, 248102 (2010).

[16] K. Morgan, D. M. Paganin, and K. K. W. Siu, Appl. Phys. Lett. 100, 124102 (2012).

[17] H. Wang, Y. Kashyap, and K. Sawhney, Phys. Rev. Lett. 114, 103901 (2015)

[18] W. Yashiro, Y. Terui, K. Kawabata, and A. Momose, Opt. Express 18, 16890 (2010).

[19] D. E Silva, Appl. Opt. 11, 2613 (1972).

[20] S. Cartier, M. Kagias, Z. Wang, A. Bergamaschi, R. Dinapoli, A. Mozzanica, B. Schmitt, and M. Stampanoni (unpublished).

[21] See Supplemental Material at http://link.aps.org/ supplemental/10.1103/PhysRevLett.116.093902 for the derivation of Eq. (7), the absorption, differential phase in $\mathrm{x}$ and $\mathrm{y}$ of the carbon fiber loop, and a video containing the individual scattering images of the carbon fiber loop.

[22] K. Morgan, D. Paganin, and K. K. W. Siu, Opt. Express 19, 19781 (2011)

[23] I. Zanette, T. Zhou, A. Burvall, U. Lundström, D. H. Larsson, M. Zdora, P. Thibault, F. Pfeiffer, and H. M. Hertz, Phys. Rev. Lett. 112, 253903 (2014).

[24] M. Stampanoni, A. Groso, A. Isenegger, G. Mikuljan, Q. Chen, A. Bertrand, S. Henein, R. Betemps, U. Frommherz, P. Bohler, D. Meister, M. Lange, and R. Abela, AIP Conf. Proc. 879, 848 (2007).

[25] T. H. Jensen, M. Bech, O. Bunk, T. Donath, C. David, R. Feidenhans'1, and F. Pfeiffer, Phys. Med. Biol. 55, 3317 (2010).

[26] M. Strobl, Sci. Rep. 4, 7243 (2014).

[27] C. David, J. Bruder, T. Rohbeck, C. Grünzweig, C. Kottler, A. Diaz, O. Bunk, and F. Pfeiffer, Microelectron. Eng. 84, 1172 (2007).

[28] S. Rutishauser, M. Bednarzik, I. Zanette, T. Weitkamp, M. Börner, J. Mohr, and C. David, Microelectron. Eng. 101, 12 (2013).

[29] S. Cartier, A. Bergamaschi, R. Dinapoli, D. Greiffenberg, I. Johnson, J. H. Jungmann, D. Mezza, A. Mozzanica, B. Schmitt, X. Shi, M. Stampanoni, J. Sun, and G. Tinti, J. Instrum. 9, C05027 (2014). 